\title{
Understanding Mechanisms of Genetic Risk for Adolescent Internalizing and Externalizing Problems: The Mediating Role of Parenting and Personality
}

\author{
Jinni Su, ${ }^{1}$ Sally I-Chun Kuo, ${ }^{1}$ Kathleen K. Bucholz, ${ }^{2}$ Howard J. Edenberg, ${ }^{3}$ John R. Kramer, ${ }^{4}$ \\ Marc Schuckit, ${ }^{5}$ and Danielle M. Dick ${ }^{1,6}$ \\ ${ }^{1}$ Department of Psychology, Virginia Commonwealth University, Richmond, Virginia, USA \\ ${ }^{2}$ Department of Psychiatry, Washington University School of Medicine in St. Louis, St. Louis, Missouri, USA \\ ${ }^{3}$ Department of Biochemistry and Molecular Biology, Indiana University, Bloomington, Indiana, 47405, USA \\ ${ }^{4}$ Department of Psychiatry, University of lowa, lowa City, lowa, USA \\ ${ }^{5}$ Department of Psychiatry, University of California at San Diego, La Jolla, California, USA \\ ${ }^{6}$ Department of Human and Molecular Genetics, Virginia Commonwealth University, Richmond, Virginia, USA
}

\begin{abstract}
Genetic predispositions play an important role in the development of internalizing and externalizing behaviors. Understanding the mechanisms through which genetic risk unfolds to influence these developmental outcomes is critical for developing prevention and intervention efforts, capturing key elements of Irv's research agenda and scientific legacy. In this study, we examined the role of parenting and personality in mediating the effect of genetic risk on adolescents' major depressive disorder and conduct disorder symptoms. Longitudinal data were drawn from a sample of 709 European American adolescents and their mothers from the Collaborative Studies on Genetics of Alcoholism. Results from multivariate path analysis indicated that adolescents' depressive symptoms genome-wide polygenic scores (DS_GPS) predicted lower parental knowledge, which in turn was associated with more subsequent major depressive disorder and conduct disorder symptoms. Adolescents' DS_GPS also had indirect effects on these outcomes via personality, with a mediating effect via agreeableness but not via other dimensions of personality. Findings revealed that the pattern of associations was similar across adolescent gender. Our findings emphasize the important role of evocative gene-environment correlation processes and intermediate phenotypes in the pathways of risk from genetic predispositions to complex adolescent outcomes.
\end{abstract}

Keywords: genes, parenting, personality, depression, conduct problems

Internalizing and externalizing problems are prevalent among adolescents and are associated with a host of negative psychosocial outcomes, such as poor school performance, impaired social functioning, increased risk for substance use and misuse, and other comorbid psychiatric disorders (Frojd et al., 2008; Rhew et al., 2017; Rohde et al., 2013; Small et al., 2008). According to the National Comorbidity Study, lifetime prevalence rates of major depressive disorders and conduct disorders were $11.7 \%$ and $6.8 \%$, respectively, among adolescents aged 13-18 (Merikangas et al., 2010). Genetic predispositions play an important role in the development of these internalizing and externalizing behaviors (Burmeister et al., 2008; Lohoff, 2010). Very little is known, however, about how genetic factors affect these complex behaviors. Understanding the mechanisms through which genetic risk unfolds to influence adolescent internalizing and externalizing problems is critical for developing prevention and intervention efforts. This article represents not just a tribute, but an application of Irv Gottesman's lively research agenda and scientific legacy.

Individuals' genetic predispositions may affect their psychiatric and behavioral outcomes through mechanisms at multiple levels (e.g., individual, family), as risk and

\section{RECEIVED 5 April 2018; ACCEPTED 4 May 2018}

ADDRESS FOR CORRESPONDENCE: Jinni Su, PhD, Department of Psychology, Virginia Commonwealth University, Richmond, 23284 VA, USA.E-mail: jsu2@vcu.edu 
protective factors at different levels play important roles in influencing adolescent adjustment. Two plausible mechanisms are parenting (through gene-environment correlation processes) and personality (as intermediate phenotypes), both of which have been shown to be genetically influenced (Klahr \& Burt, 2014; Vukasovic \& Bratko, 2015) and to affect adolescent internalizing and externalizing problems (Castellanos-Ryan et al., 2016; Goldner et al., 2016). In this study, we examine parenting and personality dimensions simultaneously as important mediating pathways for genetic influences on adolescent internalizing (as indexed by major depressive disorder symptoms) and externalizing (as indexed by conduct disorder symptoms) problems. Given that internalizing and externalizing problems often co-occur (Wolff \& Ollendick, 2006) and have shared genetic underpinings (Cosgrove et al., 2011; Subbarao et al., 2008), we take a multivariate approach to examining whether and how adolescents' genetic predispositions may influence their development of major depressive disorder (MDDSX) and conduct disorder symptoms (CDSX) directly and/or indirectly through parenting and personality.

\section{Parenting as a Mechanism for Genetic Influences Via Gene-Environment Correlation Processes}

Parenting is a key environmental factor that influences adolescent development, and parenting behaviors, such as monitoring and knowledge, and support and warmth have been associated with lower risk for depression, conduct disorder, subclinical internalizing and externalizing problems, and related psychosocial outcomes among adolescents (Frojd et al., 2007; Hamza \& Willoughby, 2011; Racz \& McMahon, 2011). Theory and research suggest that children's genetic predispositions play important roles in influencing parenting behavior (Avinun \& Knafo, 2014) and that individuals shape their environments and experiences through gene-environment correlation processes (Plomin et al., 1977; Scarr \& McCartney, 1983). It is also well demonstrated that genetic factors contribute to variations in parenting behaviors. Findings from a meta-analysis indicated that heritability of parenting behaviors ranges from $23 \%$ to $40 \%$ (Klahr \& Burt, 2014). Twin and adoption studies have indicated that both parents' and children's genetic predispositions contribute to variations in parenting behaviors (Klahr \& Burt, 2014; McGuire et al., 2012; Neiderhiser et al., 2004), providing support for both passive and evocative gene-environment correlations. Passive gene-environment correlation refers to the association between parents' genotypes and the environments (e.g., parenting behaviors) they provide for their children; evocative gene-environment correlation occurs when children's genetic predispositions evoke environmental responses (Scarr \& McCartney, 1983). There is also evidence from studies of measured genotypes that both mothers' and children's genotypes are associated with maternal parenting behavior (Leerkes et al., 2017; van IJzendoorn et al., 2008). Thus, gene-environment correlation processes in relation to parenting have been demonstrated in both latent and measured genetic studies.

Behavioral genetic research also finds significant overlap in genetic influences between parenting and offspring's psychiatric outcomes, again pointing to the importance of gene-environment correlation processes in the context of parenting and adolescent psychopathology. For example, in a sample of adolescent sibling pairs, the association between mother-child conflict and subsequent adolescent major depressive disorder symptoms was explained predominantly by common genetic influences (Samek et al., 2016). Taken together, these suggest that gene-environment correlation processes related to parenting may serve as a mechanism by which genetic factors influence adolescent psychiatric outcomes.

In this study, we examine whether adolescents' genetic predispositions affect parental knowledge, which in turn influence adolescents' MDDSX and CDSX. Parental knowledge is defined here as the degree to which parents know about their offspring's activities, interests, and whereabouts, which is partly influenced by parental monitoring and offspring disclosure, and may reflect the overall quality of parent-child relationship (Kerr \& Stattin, 2000). We focus on parental knowledge because it is a commonly studied dimension of parenting that has shown robust associations with a host of adolescent psychosocial outcomes (Kelly et al., 2017; Lac \& Crano, 2009; Racz \& McMahon, 2011).

\section{Personality Dimensions as Intermediate Phenotypes Linking Genetic Influences to Adolescent Outcomes}

Because complex behavioral outcomes such as MDDSX and CDSX are quite distal from the level of gene function, studying the mediating role of intermediate phenotypes (sometimes also referred to as endophenotypes) may help to delineate the mechanisms through which genetic risks unfold across development to affect psychiatric and behavioral outcomes (Gottesman \& Gould, 2003; Lenzenweger, 2013). Personality domains are compelling intermediate phenotypes for molecular genetic studies of complex psychiatric and behavioral outcomes. First, personality domains are moderately to strongly heritable (Vukasovic \& Bratko, 2015); twin and adoption studies indicate that heritability estimates range between $40 \%$ and $60 \%$ for different personality domains, such as extraversion and neuroticism (Bouchard \& McGue, 2003; Vukasovic \& Bratko, 2015). Second, personality domains are robustly associated with major depressive disorder and related psychiatric outcomes (Klein et al., 2011). For example, neuroticism is associated with higher risk for major depressive disorder and conduct/externalizing disorder among adolescents and adults (Kotov et al., 2010). Third, personality domains are relatively stable traits (Roberts et al., 2001) that co-segregate with major depressive disorder and related outcomes. For example, siblings from families with a currently depressed 
proband reported higher levels of neuroticism than those from families without any history of depression (Farmer et al., 2002). Furthermore, twin studies suggest significant overlap between genetic factors contributing to personality and major depressive disorder (Fanous et al., 2002) and related psychiatric outcomes, such as conduct disorder (Blonigen et al., 2005).

Taken together, there is compelling evidence identifying personality domains as intermediate phenotypes for molecular genetic studies of psychiatric and behavioral outcomes such as internalizing and externalizing problems. Indeed, neuroticism has been suggested as a promising endophenotype for depression (Goldstein \& Klein, 2014). However, very few studies have explicitly investigated the role of these putative intermediate phenotypes in the gene-behavior pathway to psychopathology (Lenzenweger, 2013). Personality is a multi-dimensional construct, and multiple dimensions of personality have been associated with a host of psychosocial outcomes (Klein et al., 2011; Kotov et al., 2010). Recently, Li and colleagues (2017) examined the role of sensation seeking and the Big Five personality traits (i.e., neuroticism, extraversion, agreeableness, openness to experience, and conscientiousness) in mediating genetic influences on alcohol use problems. They argued that because personality dimensions tend to "hang together", studies need to examine their effects simultaneously in order to evaluate the unique effect of each personality domain (Li et al., 2017). In this study, we adopt a similar approach to examine the role of adolescents' Big Five personality traits (in addition to parental knowledge) in mediating the effect of adolescents' genetic predisposition on their MDDSX and CDSX.

\section{The Present Study}

The goal of this study was to examine the role of parental knowledge and adolescents' Big Five personality domains (i.e., neuroticism, extraversion, agreeableness, openness to experience, and conscientiousness) in mediating the effects of adolescents' genetic predispositions toward depression related to the manifestation of MDDSX and CDSX. Parenting and personality are both potentially important pathways through which genetic risk may impact adolescent outcomes. Previous genetically informed studies have tended to examine the role of parenting and personality in relation to adolescent outcomes separately (e.g., Elam et al., 2017; Li et al., 2017). This is important to note, given that parenting and adolescent personality are reciprocally related (de Haan et al., 2012; Schofield et al., 2012) so that considering them in separate analyses may hinder the understanding of the unique effects of each pathway. Here, we extend the literature to test these mediating pathways simultaneously in one model in order to evaluate their unique effects.

Complex behavioral outcomes such as major depressive disorder and conduct disorder are polygenic such that many common genetic variants of small effects contribute to risk (Hyde et al., 2016). In this study, we index adolescents' genetic predispositions by considering the aggregate effects of common genetic variants (i.e., genome-wide polygenic scores) that impact depressive symptoms. This approach of genome-wide polygenic scoring has been used to predict risk for major depressive disorder (Mullins et al., 2016; Peyrot et al., 2014), externalizing disorders (Salvatore et al., 2015), and other related outcomes (Bogdan et al., 2018). The calculation of reliable polygenic scores requires a discovery genome-wide association study (GWAS) with an extremely large sample size to estimate the effects of common genetic variants across the genome (Dudbridge, 2013; Martin et al., 2017). We focus on depressive symptoms genomewide polygenic scores (DS_GPS) in this study because the GWAS on depressive symptoms conducted by the Social Science Genetic Association Consortium (SSGAC; Okbay et al., 2016) has one of the largest sample sizes to date, providing us the opportunity to calculate relatively reliable DS_GPS. No parallel large-scale analysis exists for conduct disorder; thus, reliable, predictive polygenic scores for this outcome are not yet available for study.

Given that prior research suggests overlap in genetic influences on major depressive disorder and conduct disorder (Subbarao et al., 2008), we hypothesized that adolescents' DS_GPS would influence both their MDDSX and CDSX. Further, we hypothesized that these genetic effects would, at least in part, manifest indirectly by influencing parental knowledge and personality domains. Since prior research suggests that both mothers' and children's genotypes can affect parenting behaviors, we took into account mothers' DS_GPS, in addition to adolescents' DS_GPS, to delineate whether genetic effects on parental knowledge represent passive or evocative gene-environment correlations. We also tested adolescents' gender as a moderator of the hypothesized pathways, given evidence for gender differences in the rates of internalizing and externalizing problems (Kessler et al., 2005; Merikangas et al., 2010; Nock et al., 2006); note that our sample was approximately evenly split between males and females. Our examination of gender differences in pathways of risk is exploratory and thus no specific hypotheses were proposed.

\section{Materials and Methods}

\section{Sample}

Data were drawn from the Collaborative Studies on Genetics of Alcoholism (COGA) Prospective Study. COGA is a multi-site, large family study that aims to identify genes involved in alcohol dependence and related psychiatric phenotypes (Begleiter et al., 1995). The Prospective Study sample is a subset of the larger COGA study, focused on understanding how genetic risk unfolds across adolescence into young adulthood (Dick et al., 2014). COGA families were identified through probands in inpatient or outpatient 
alcohol treatment programs at six U.S. sites. Additional 'community comparison' families were obtained through a variety of sources such as driver's license registries and dental clinics. Recruitment for the Prospective Study began in December 2004 among offspring of the original COGA families. Recruited participants had at least one parent who was interviewed in one of the previous phases of COGA. Participants in the COGA Prospective Study were interviewed at enrollment and followed up at approximately biennial intervals. The Institutional Review Boards at all sites approved this study, and written consent (and assent for adolescents) was obtained from all participants.

The present sample included adolescents (aged 12-17 years) from the COGA Prospective Study and their mothers. We included adolescents who (1) had genomic data available, (2) had completed their baseline assessment at enrollment and first follow-up assessment (approximately two years after the baseline), (3) were under 18 years of age at their first follow-up assessment, and (4) were of European ancestry as determined by genetic ancestry principal component analysis. We focused on European American adolescents because the sample of the discovery GWAS was primarily of European descent (Okbay et al., 2016), and genome-wide polygenic scores have better prediction accuracy when the discovery and target samples match in terms of ancestry (Martin et al., 2017). The strategy resulted in an analytic sample of 709 adolescents (49.4\% female) from 336 COGA extended families. This is a relatively early adolescent sample, as $46.5 \%$ of the sample was aged 12 years at their baseline assessment $\left(M_{\text {age }}=13.01, S D=1.14\right)$. The adolescent version of the Semi-Structured Assessment for the Genetics of Alcoholism (C-SSAGA; Bucholz et al., 1994), a comprehensive interview that assesses alcohol use disorders and other psychiatric phenotypes such as major depressive disorder and conduct disorder, was administered by trained interviewers in adolescents' homes at baseline and at each follow-up assessment. The majority of the adolescents' mothers (565 or $79.7 \%$ ) provided DNA samples in the COGA adult sample and had genomic data available.

\section{Measures}

Parental knowledge. Adolescents answered three questions (how much their parental figures know about their plans, their interests, and where and with whom they spend time when not at home) from Chassin et al. (1993), as part of C-SSAGA at baseline. Responses were made on a 4-point scale from 1 (rarely) to 4 (always), and the inter-item correlations ranged from 0.45 to 0.53 (all $p<.0001$ ). Items were averaged and higher scores indicated higher parental knowledge. Cronbach's alpha for this scale was 0.73 . Some adolescents $(n=29,4.1 \%)$ reported parental knowledge of non-biological mothers because they did not live with their biological mothers at the time of assessment. For the pur- pose of this study, we coded those responses as missing and only used data reported on cohabitating biological mothers.

Personality domains. Adolescents completed the reliable and well-validated NEO Five-Factor Inventory (Costa \& McCrae, 1992) at baseline. This measure includes 60 items that assess five domains of personality (12 items for each dimension). All items were rated on a 5-point scale from 1 (strongly disagree) to 5 (strongly agree). The five personality domains are extraversion (e.g., 'I like to have a lot of people around me'), openness to experience (e.g., 'I often try new and foreign foods'), agreeableness (e.g., 'I am a cheerful, high-spirited person'), conscientiousness (e.g., 'when I make a commitment, I can always be counted on to follow through'), and neuroticism (e.g., 'I tend to be cynical and skeptical of others' intentions').

Major depressive disorder symptoms (MDDSX). Clinical criterion counts for major depressive disorders were obtained from C-SSAGA at adolescents' first follow-up interview (approximately two years after the baseline assessment). MDDSX was operationalized as the number of major depressive disorder symptoms (e.g., depressed mood or irritable most of the day, nearly every day) ever endorsed according to the Diagnostic and Statistical Manual of Mental Disorders (DSM-IV; American Psychiatric Association, 1994 ) and had a possible range of $0-9$. Because $80 \%$ of the adolescents endorsed zero MDDSX, we recoded MDDSX to a dichotomous variable to indicate whether or not adolescents had any $\operatorname{MDDSX}(1=$ yes, $0=$ no $)$.

Conduct disorder symptoms (CDSX). Clinical criterion counts for conduct disorders were obtained from C-SSAGA at adolescents' first follow-up interview. CDSX was operationalized as the number of conduct disorder symptoms (e.g., often bullies, threatens, or intimidates others) ever endorsed according to the DSM-IV (American Psychiatric Association, 1994) and had a possible range of $0-15$. In the current study, adolescents' CDSX ranged from 0 to 10 , with $64 \%$ of the adolescents endorsing zero CDSX, $18.9 \%$ endorsed 1 CDSX, 9.2\% endorsing 2 CDSX, and 7.9\% endorsing 3 or more CDSX. For the purpose of the current study, we grouped adolescents into four categories to indicate their conduct problems $(0=$ no CDSX, $1=$ one CDSX, $2=$ two CDSX, $3=$ three or more CDSX).

Genotyping and depressive symptoms genome-wide polygenic scores (DS_GPS). Adolescents' and their mothers' DNA samples were genotyped using the Illumina $2.5 \mathrm{M}$ array (Illumina, San Diego, CA, USA) or the smokescreen array (Baurley et al., 2016). Data were imputed to 1000 Genome, and single nucleotide polymorphisms (SNPs) with a genotyping rate $<0.95$ or that violated Hardy-Weinberg equilibrium $\left(p<10^{-6}\right)$ or with minor allele frequency $(\mathrm{MAF})<0.01$ were excluded from analysis. 


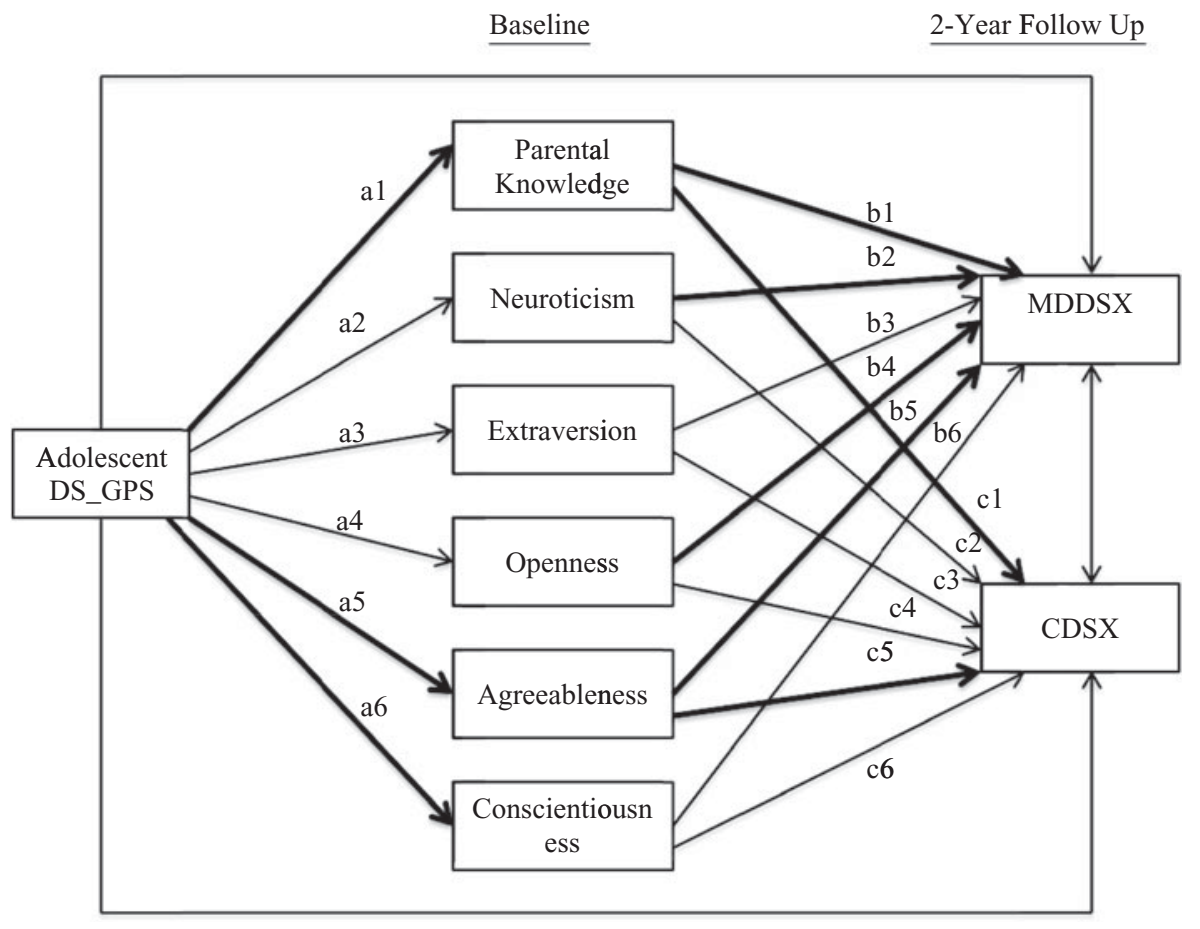

\section{FIGURE 1}

Path model predicting adolescent internalizing and externalizing problems from depressive symptoms polygenic scores via parental knowledge and personality. Adolescents' age, sex, and genetic ancestry principal components were included as covariates for parental knowledge, personality domains, and adolescent outcomes. Mothers' depressive symptoms genome-wide polygenic score was also included as covariate for parental knowledge and allowed to correlate with adolescents' DS_GPS. Paths for covariates are not shown in the figure. Statistically significant paths are bolded. DS_GPS = depressive symptoms polygenic scores; MDDSX = major depressive disorder symptoms; CDSX = conduct disorder symptoms.

We used genome-wide association estimates for depressive symptoms from the SSGAC (Okbay et al., 2016), the largest published GWAS of depressive symptoms to date, to calculate depressive symptoms genome-wide polygenic scores (DS_GPS) for adolescents and their mothers in our sample.

We used the - score procedure in PLINK (Purcell et al., 2007), which computes a linear function of the number of scored alleles an individual possesses weighted by the associated GWAS $t$ statistic. SNPs were pruned for linkage disequlibrium (LD), based on 1,000 Genome phase 3 reference panel genotype data with clumping based on SSGAC GWAS $p$-values, using a $500 \mathrm{~kb}$ physical distance and an LD threshold of $r^{2} \geq 0.25$. Given that there are no set criteria for establishing a threshold to create maximally informative scores (Evans et al., 2009), we calculated a series of polygenic scores in our sample that included SNPs meeting decreasingly stringent $p$-value thresholds $(p<.0001, p<.001$, $p<.01, p<.10, p<.20, p<.30, p<.40, p<.50)$ in the SSGAC discovery GWAS (Okbay et al., 2016).

\section{Analysis}

Preliminary analyses were first conducted to determine which $p$-value threshold to use for adolescents' depres- sive symptoms genome-wide polygenic scores (DS_GPS) in subsequent analyses. Descriptive statistics and intercorrelations between study variables were also examined. Next, path analysis was conducted using Mplus version 7.3 to examine the direct and indirect effect of adolescents' DS_GPS on adolescent major depressive disorder (MDDSX) and conduct disorder symptoms (CDSX), via parental knowledge and adolescent personality domains. Figure 1 provides a graphical illustration of the path model. In this model, parental knowledge and adolescents' Big Five domains of personality were modeled simultaneously as mediators of the effect of adolescents' DS_GPS on adolescent MDDSX and CDSX. Specifically, adolescents' DS_GPS was specified as an exogenous variable associated with parental knowledge and personality domains, which in turn were specified to be associated with adolescent MDDSX and CDSX. Adolescents' DS_GPS was also specified to be directly associated with adolescent MDDSX and CDSX. Adolescent MDDSX and CDSX were also specified to be correlated, as were the different personality domains.

Given prior findings from the literature that parenting is associated with adolescent personality (de Haan et al., 2012), we also specified parental knowledge to be correlated 
with personality domains in the model. Adolescents' age and sex were included as covariates for parental knowledge, personality domains, and adolescent outcomes, along with the first three genetic ancestry principal components to account for potential population stratification. To account for potential passive gene-environment correlation, mothers' DS_GPS was included as a covariate for parental knowledge. Adolescents' and mothers' DS_GPS were specified to be correlated.

Indirect effects of adolescents' DS_GPS on adolescent outcomes via parental knowledge and personality domains were tested using the MODEL INDIRECT command in Mplus, which provides a test of specific indirect effects in addition to the total indirect and direct effects of DS_GPS on adolescent outcomes. Given that multiple indirect pathways are examined simultaneously, specific indirect effects reflect each of the specific pathways (e.g., adolescent DS_GPS $\rightarrow$ parental knowledge $\rightarrow$ MDDSX) while also accounting for the shared associations between them. Indirect effects were evaluated using bias-corrected bootstrapping (1,000 times) 95\% confidence interval (CI; MacKinnon et al., 2004), with CI not including zero indicating statistically significant indirect effects. Clustering within families was taken into account using the CLUSTER command in Mplus. Missing data were accounted for using full information maximum likelihood (FIML).

To examine the possible differences in path coefficients across adolescent gender, multigroup analysis was conducted by removing gender from the path model and then comparing a model with all remaining paths constrained to equality with one that had all paths freely estimated across males and females. A statistically significant Wald chi-square test of parameter equalities would indicate significant differences in path coefficients across gender.

\section{Results}

\section{Preliminary Analyses}

We conducted linear regressions to predict adolescents' MDDSX and CDSX from adolescents' DS_GPS calculated using nominally associated sets of SNPs from each of the $p$ value thresholds (Table 1). Adolescents' DS_GPS calculated from SNPs at $p<.01$ or below were generally not associated with these adolescent outcomes, but those calculated from SNPs at $p<.05$ and above were positively predictive of adolescents' MDDSX and/or CDSX. Adolescents' DS_GPS at $p<.20$ and above explained approximately $1.2 \%$ and $1.0 \%$ of the phenotypic variance in MMDSX and CDSX, respectively, above and beyond the effects of the covariates (i.e., adolescents' age, sex, and genetic ancestry principal components). We conducted subsequent analyses using the threshold at $p<.20$ for adolescents' DS_GPS, as this was the most parsimonious polygenic score (with the fewest SNPs) that predicted adolescent MDDSX and CDSX. We used the same $p$-value threshold for mothers' DS_GPS.
Descriptive statistics and correlations between variables are presented in Table 2 . It appeared that $20 \%$ of the adolescents endorsed at least one MDDSX, and 64\%, 18.9\%, $9.2 \%$, and $7.9 \%$ of adolescents endorsed $0,1,2$, and 3 CDSX, respectively, $(M=0.64, S D=0.91)$. Adolescents' DS_GPS was significantly correlated with parental knowledge, agreeableness, and conscientiousness, in addition to adolescent MDDSX and CDSX; parental knowledge and personality domains were generally correlated with adolescent MDDSX and CDSX, confirming the promise of examining parenting and personality as mediators for the effect of adolescents' DS_GPS on adolescent outcomes. MDDSX and CDSX were moderately correlated with each other, suggesting that they are related but distinct indicators of adolescent maladjustment.

\section{Predicting Major Depressive Disorder and Conduct Disorder Symptom Counts}

The path model demonstrated excellent fit $\left(\chi^{2}=18.42\right.$, $d f=13, p=.14$, CFI $=0.99$, RMSEA $=0.02)$. Results from the path model predicting adolescents' major depressive disorder (MDDSX) and conduct disorder symptom counts (CDSX) are summarized in Table 3. Consistent with expectation, adolescents' DS_GPS was associated with lower parental knowledge; however, mothers' DS_GPS was not associated with parental knowledge. Adolescents' DS_GPS was associated with lower agreeableness and conscientiousness, but was not associated with neuroticism, extraversion, and openness to experience. Parental knowledge and adolescents' agreeableness were associated with lower adolescent MDDSX and CDSX. Adolescents' neuroticism and openness to experience were associated with higher MDDSX, but were not associated with CDSX. Adolescents' extraversion and conscientiousness were not associated with their CDSX or MDDSX.

Consistent with our hypothesis, results indicated that there were significant indirect effects of adolescents' DS_GPS on MDDSX and CDSX via parental knowledge and personality. Coefficients and confidence intervals for the total and specific indirect effects are presented in Table 3. Specifically, adolescents' DS_GPS was associated with higher adolescent MDDSX and CDSX indirectly via undermining parental knowledge. Adolescent DS_GPS was also associated with higher MDDSX and CDSX indirectly via lower adolescent agreeableness. There was no significant indirect effect of adolescents' DS_GPS on adolescent MDDSX and CDSX through neuroticism, extraversion, openness to experience, and conscientiousness. There was also no evidence that DS_GPS was associated with MDDSX and CDSX, independent of its effect on parental knowledge and personality domains.

\section{Examining Gender Differences in Pathways of Risk}

Results from the multigroup analyses indicated that there were no significant gender differences in path coefficients 


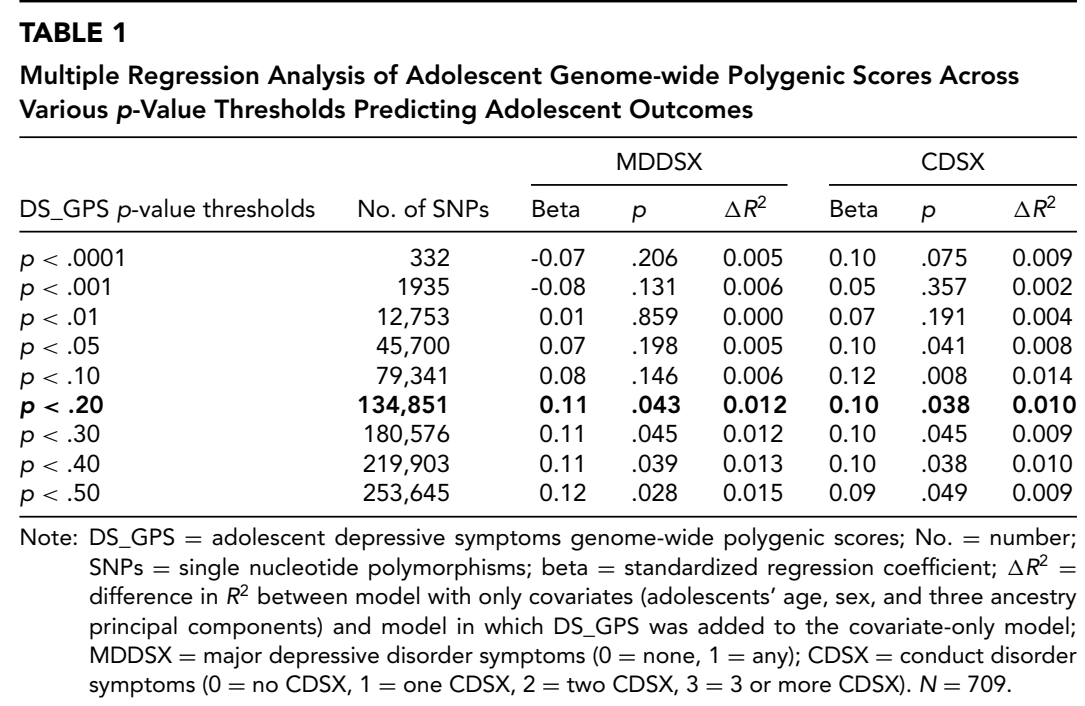

TABLE 2

Descriptive Statistics and Bivariate Correlations Between Variables

\begin{tabular}{|c|c|c|c|c|c|c|c|c|c|c|c|c|c|}
\hline & $\mathrm{N}$ & 1 & 2 & 3 & 4 & 5 & 6 & 7 & 8 & 9 & 10 & 11 & 12 \\
\hline 1. Age & 709 & - & & & & & & & & & & & \\
\hline 2. Sex & 709 & 0.01 & - & & & & & & & & & & \\
\hline 3. ADS_GPS & 709 & 0.03 & -0.05 & - & & & & & & & & & \\
\hline 4. M DS_GPS & 565 & -0.04 & -0.06 & 0.56 & - & & & & & & & & \\
\hline 5. Par knowledge & 707 & -0.15 & -0.13 & -0.08 & -0.02 & - & & & & & & & \\
\hline 6. Neuroticism & 647 & 0.02 & 0.08 & 0.04 & -0.01 & -0.24 & - & & & & & & \\
\hline 7. Extraversion & 647 & -0.04 & -0.03 & -0.06 & -0.02 & 0.16 & -0.13 & - & & & & & \\
\hline 8. Openness & 647 & 0.04 & -0.15 & -0.04 & 0.06 & -0.01 & 0.13 & 0.13 & - & & & & \\
\hline 9. Agreeableness & 647 & -0.07 & 0.01 & -0.14 & 0.03 & 0.34 & -0.39 & 0.31 & 0.09 & - & & & \\
\hline 10. Conscientiousness & 647 & 0.00 & -0.06 & -0.11 & -0.02 & 0.35 & -0.31 & 0.36 & 0.07 & 0.54 & - & & \\
\hline 11. MDDSX & 709 & 0.11 & -0.13 & 0.09 & 0.05 & -0.22 & 0.25 & -0.09 & 0.16 & -0.24 & -0.13 & - & \\
\hline 12. CDSX & 709 & -0.03 & 0.12 & 0.07 & 0.03 & -0.33 & 0.13 & -0.08 & -0.01 & -0.33 & -0.23 & 0.22 & - \\
\hline M & & 13.01 & $0.51^{a}$ & -0.00 & -0.00 & 3.48 & 50.86 & 56.28 & 45.48 & 46.57 & 42.32 & $0.20^{\mathrm{b}}$ & 0.64 \\
\hline$S D$ & & 1.14 & - & 0.00 & 0.00 & 0.58 & 10.25 & 10.28 & 9.36 & 11.51 & 11.21 & - & 0.91 \\
\hline
\end{tabular}

Note: $\mathrm{A}$ = adolescent; $\mathrm{M}=$ mother; DS_GPS = depressive symptoms polygenic scores; Par = parental; MDDSX = major depressive disorder symptom count; $\mathrm{CDSX}=$ conduct disorder symptom count; All bolded coefficients are statistically significant at $p<.05$. Sex was coded $1=$ male and $0=$

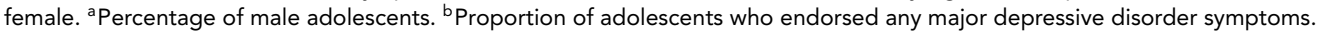

in the model predicting MDDSX and CDSX $\left(\chi^{2}=21.78\right.$ $d f=21, p=.41$ ), suggesting that the pathways linking adolescents' DS_GPS to adolescents' MDDSX and CDSX via parental knowledge and personality were similar for males and females. Detailed results are not presented, but are available upon request.

\section{Discussion}

The overarching goal of this study was to examine the role of parenting and personality in mediating the effect of genetic risk on adolescents' internalizing and externalizing problems. Our findings revealed that adolescents' DS_GPS predicted lower parental knowledge, which in turn was associated with more subsequent MDDSX and CDSX. In addition, findings indicated that the effects of adolescents' DS_GPS on these outcomes were also indirect via personality, with a mediating effect via agreeableness, but not other dimensions of personality. Findings revealed that the pattern of associations was similar across gender.

It is well known that parenting plays an important role in adolescent development and that genes and environment function in concert to influence psychosocial outcomes (Horwitz \& Neiderhiser, 2011). Gene-environment interplay processes are complex and can come in different forms, such as gene-environment interaction and gene-environment correlation. Numerous behavioral genetic studies have demonstrated that parenting behaviors interact with genetic factors to influence child and adolescent outcomes. In particular, parental monitoring and knowledge have been shown to moderate genetic influences on adolescent outcomes such as substance use and externalizing problems across studies using different genetic approaches (i.e., candidate genes, polygenic risk scores, twin studies; Cooke et al., 2015; Salvatore et al., 2015). Our findings extend the literature to provide evidence that, in addition to functioning as a moderator of genetic 


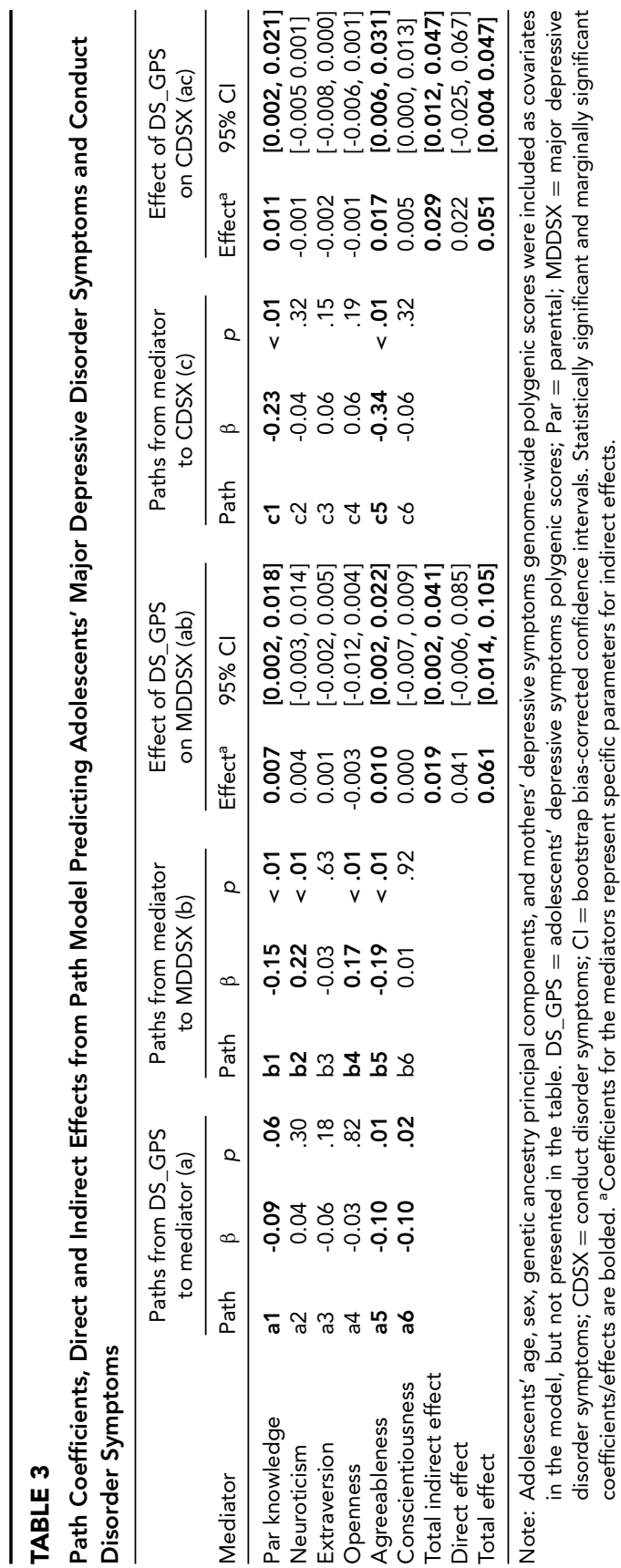

influences through gene-environment interaction processes as demonstrated in prior research, parenting also serves as a mediator for genetic influences on adolescent outcomes through gene-environment correlation processes. Specifically, adolescents' genetic risk for depressive symptoms evokes lower levels of parental knowledge, likely indicative of lower adolescent disclosure and poorer quality of parent-child relationship overall; this, in turn, is associated with adverse adolescent outcomes such as major depressive disorder and conduct disorder symptoms. These genetic effects are apparent after controlling for mothers' genetic risk for depressive symptoms (which accounted for passive gene-parenting effects), thus indicative of evocative gene-parenting effects. Although gene-environment correlations in relation to parenting have been demonstrated in prior research (Avinun \& Knafo, 2014), our findings indicate that evocative gene-environment correlations, particularly those related to family processes, are important mechanisms by which genetic risk impacts adolescent psychosocial outcomes (Elam et al., 2017).

Personality also plays an important role in individual differences in psychiatric outcomes (Andersen \& Bienvenu, 2011). Our findings indicated that all of the Big Five personality domains were correlated with adolescents' internalizing and/or externalizing problems as indexed by major depressive disorder and conduct disorder symptoms. However, in the multivariate path model in which the effects of parental knowledge and personality domains were considered simultaneously, only agreeableness was associated with both lower adolescent MDDSX and CDSX. Neuroticism and openness to experience were associated with higher MDSX, but extraversion and conscientiousness were unrelated. Furthermore, supporting the notion that personality dimensions serve as intermediate phenotypes for genetic effects on psychiatric outcomes (Goldstein \& Klein, 2014; Li et al., 2017), our findings indicated that agreeableness, but not the other Big Five personality dimensions, mediated the effect of genetic risk for depressive symptoms on adolescents' MDDSX and CDSX.

The foregoing findings are consistent with prior findings suggesting the important role of agreeableness in adolescent psychosocial adjustment. For example, Jensen-Campbell and colleagues (2002) found that of the Big Five personality dimensions, agreeableness was most closely associated with adolescents' psychosocial outcomes, such as peer relations and aggression (Gleason et al., 2002). Given that agreeableness is associated with motives to maintain positive interpersonal relations (Jensen-Campbell \& Graziano, 2001) and that maintaining good quality peer relationships is of particular importance for psychosocial adjustment during adolescence (Brown \& Larson, 2009), it is not surprising that agreeableness exerted an important role in influencing and mediating genetic effects on adolescent outcomes in our sample. Perhaps agreeableness is particularly influential for adolescents' psychosocial outcomes by affecting 
their peer relationships and social functioning. Future research is needed to explicitly test the mechanisms through which personality domains influence adolescent outcomes.

It is interesting that extraversion and conscientiousness were not associated with adolescent MDDSX and CDSX in our sample when parental knowledge and other Big Five personality dimensions were considered. This seems to be contradictory to prior findings that extraversion and conscientiousness are associated with a host of psychiatric disorders and related outcomes in adolescents and adults (Castellanos-Ryan et al., 2016; Kotov et al., 2010; Miller et al., 2008; Watson et al., 2015). We note, however, that these studies primarily focused on older adolescents or adult samples, whereas our sample included relatively younger adolescents. Given that personality traits may change across development, for example, conscientiousness increases in adulthood (Roberts et al., 2006), it is possible that the effects of personality domains on psychiatric outcomes also vary across development. Future studies using longitudinal designs with multiple assessments on personality are warranted to investigate how these effects may change from early adolescence to adulthood.

Strengths of this study include the simultaneous tests of gene-environment correlation process (i.e., parenting) and purported intermediate phenotypes (i.e., personality domains) as mediating mechanisms to delineate the pathways by which genetic influences impact adolescent outcomes using a longitudinal design. Considering multiple mediating pathways simultaneously allowed for examination of the unique effects of each specific pathway. In addition, we took a multivariate approach to consider both adolescent internalizing and externalizing problems, which allowed us to understand whether pathways of risk are similar or different across outcomes.

Several limitations of this study need to be considered in interpreting the findings. First, our measure of parental knowledge represents adolescents' overall perceptions of their parents' knowledge of their interests/activities and whereabouts and was not specific to paternal and maternal parenting behavior. We controlled for mothers' polygenic risk scores in analyses, but fathers' polygenic scores were not accounted for due to the large amount of missing data, which may not have fully eliminated potential passive geneparenting correlation effects. However, it is important to note that mothers usually assume primary caregiving roles, such that adolescents' perceptions of parental knowledge may likely be more indicative of their perceptions of maternal knowledge. We also acknowledge that adolescent-report of parental knowledge may not reflect the reality of parental knowledge. Thus, future studies need to replicate our findings using parent-report.

Second, we only considered a single dimension of adolescents' genetic risk (i.e., genetic risk for depressive symptoms). Given prior evidence of genetic overlap between depression and other psychiatric outcomes (Cosgrove et al.,
2011; Subbarao et al., 2008), it is not possible to determine whether the observed effects are unique to genetic risk for depressive symptoms. In addition to being of theoretical interest, we chose to focus on polygenic risk for depressive symptoms because it is one of the largest published GWAS on psychiatric outcomes available to date. Future research can consider multiple dimensions of genetic risk as large-scale meta-analyses for other psychiatric outcomes emerge (e.g., genetic risk for depression and externalizing disorders), and to examine how they may function together to influence various psychiatric outcomes. In addition, we note that despite being derived using estimates from the largest GWAS on depressive symptoms to date, depressive symptoms polygenic scores only accounted for a very small amount of variance in MDDSX and CDSX in our sample and thus may have limited predictive power. Despite this limited predictive power of polygenic scores, which we consider a limitation not specific to our study but to the current field of genetic research, they provide a good opportunity for us to start understanding the mechanisms through which genetic predispositions influence complex psychiatric outcomes.

Third, we focused only on parental knowledge because it is a commonly studied aspect of parenting shown to be associated with a host of adolescent psychosocial outcomes (Kelly et al., 2017; Lac \& Crano, 2009; Racz \& McMahon, 2011). However, our sample was also limited in the parenting measures that had been collected. Future research can examine the role of other parenting behaviors in mediating genetic effects on adolescent outcomes. Fourth, we only considered two candidate mediating pathways, namely parenting and personality, for the effect of genetic predispositions on adolescent outcomes. We recognize that mechanisms for genetic influence can be further understood in multiple levels of analysis. Future studies are needed to examine other mechanisms (e.g., neurophysiological intermediate phenotypes) that underlie the pathways of genetic risk to adolescent functioning. Finally, our sample included European Americans from extended families with a history of alcoholism; thus, the findings may not be generalizable to populations of other ancestral backgrounds and/or community samples.

In conclusion, our results show that adolescents' polygenic risk for depressive symptoms influences adolescents' internalizing and externalizing problems as indexed by MDDSX and CDSX. Parenting and personality serve as important mediating pathways for genetic influences on adolescent maladjustment. In particular, we found that adolescents' depressive symptoms polygenic risk scores predicted higher risk for MDDSX and CDSX indirectly via lower parental monitoring and lower agreeableness. Our findings emphasize the important role of evocative gene-environment correlation processes and intermediate phenotypes in the pathways of risk from genetic predispositions to complex adolescent outcomes. Parenting 
and personality are, therefore, important potential targets/components for prevention and intervention efforts designed to promote positive adolescent psychosocial adjustment. We trust that Irv Gottesman would appreciate our efforts.

\section{Financial Support}

This work was supported by the National Institute on Drug Abuse and the National Institute on Alcohol Abuse and Alcoholism (D.M.D., Grant numbers U10AA008401, K02AA018755).

\section{Conflict of Interest}

None.

\section{References}

American Psychiatric Association. (1994). Diagnostic and statistical manual of mental disorders (4th ed.). Washington, DC: Author.

Andersen, A. M., \& Bienvenu, O. J. (2011). Personality and psychopathology. International Review of Psychiatry, 23, 234-247.

Avinun, R., \& Knafo, A. (2014). Parenting as a reaction evoked by children's genotype: A meta-analysis of children-astwins studies. Personality and Social Psychology Review, 18, 87-102.

Baurley, J. W., Edlund, C. K., Pardamean, C. I., Conti, D. V., \& Bergen, A. W. (2016). Smokescreen: A targeted genotyping array for addiction research. BMC Genomics, 17, 145.

Begleiter, H., Reich, T., Hesselbrock, V., Porjesz, B., Ting-Kai, L., Schuckit, M. A., ... Rice, J. P. (1995). The collaborative study on the genetics of alcoholism. Alcohol Research and Health, 19, 228-236.

Blonigen, D. M., Hicks, B. M., Krueger, R. F., Patrick, C. J., \& Iacono, W. G. (2005). Psychopathic personality traits: Heritability and genetic overlap with internalizing and externalizing psychopathology. Psychological Medicine, 35, 637648.

Bogdan, R., Baranger, D. A. A., \& Agrawal, D. (2018). Polygenic risk scores in clinical psychology: Bridging genomic risk to individual differences. Annual Review of Clinical Psychology, 14, 119-157.

Bouchard, T. J. Jr., \& McGue, M. (2003). Genetic and environmental influences on human psychological differences. Journal of Neurobiology, 54, 4-45.

Brown, B. B., \& Larson, J. (2009). Peer relationships in adolescence. In R. M. Lerner \& L. Steinberg ( Eds.), Handbook of adolescent psychology (3rd ed., pp. 74-103). New York, NY: Wiley.

Bucholz, K. K., Cadoret, R., Cloninger, C. R., Dinwiddie, S. H., Hesselbrock, V. M., Nurnberger, J. I. Jr., ... Schuckit, M. A. (1994). A new, semi-structured psychiatric interview for use in genetic linkage studies: A report on the reliability of the SSAGA. Journal of Studies on Alcohol, 55, 149-158.
Burmeister, M., McInnis, M. G., \& Zöllner, S. (2008). Psychiatric genetics: Progress amid controversy. Nature Reviews Genetics, 9, 527-540.

Castellanos-Ryan, N., Brière, F. N., O’Leary-Barrett, M., Banaschewski, T., Bokde, A., Bromberg, U., ... The IMAGEN Consortium. (2016). The structure of psychopathology in adolescence and its common personality and cognitive correlates. Journal of Abnormal Psychology, 125, 10391052.

Chassin, L., Pillow, D. R., Curran, P. J., Molina, B. S. G., \& Barrera, M. (1993). Relation of parental alcoholism to early adolescent substance use: A test of 3 mediating mechanisms. Journal of Abnormal Psychology, 102, 3-19.

Cooke, M. E., Meyers, J. L., Latvala, A., Korhonen, T., Rose, R. J., Kaprio, J., ... Dick, D. M. (2015). Gene-environment interaction effects of peer deviance, parental knowledge and dtressful life events on adolescent alcohol use. Twin Research and Human Genetics, 18, 507-517.

Cosgrove, V. E., Rhee, S. H., Gelhorn, H. L., Boeldt, D., Corley, R. C., Ehringer, M. A., ... Hewitt, J. K. (2011). Structure and etiology of co-occurring internalizing and externalizing disorders in adolescents. Journal of Abnormal Child Psychology, 39, 109-123.

Costa, P. T., \& McCrae, R. R. (1992). Manual for the revised NEO personality inventory (NEO-PIR) and NEO five-factor inventory (NEO-FFI). Odessa, FL: Psychological Assessment Resources.

de Haan, A. D., Deković, M., \& Prinzie, P. (2012). Longitudinal impact of parental and adolescent personality on parenting. Journal of Personality and Social Psychology, 102, 189-199.

Dick, D. M., Cho, S. B., Latendresse, S. J., Aliev, F., Nurnberger, J. I. Jr., Edenberg, H. J., ... Kuperman, S. (2014). Genetic influences on alcohol use across stages of development: GABRA2 and longitudinal trajectories of drunkenness from adolescence to young adulthood. Addiction Biology, $19,1055-1064$.

Dudbridge, F. (2013). Power and predictive accuracy of polygenic risk scores. PLOS Genetics, 9, e1003348.

Elam, K. K., Chassin, L., Lemery-Chalfant, K., Pandika, D., Wang, F. L., Bountress, K., ... Agrawal, A. (2017). Affiliation with substance-using peers: Examining gene-environment correlations among parent monitoring, polygenicrisk, and children's impulsivity. Developmental Psychobiology, 59, 561-573.

Evans, D. M., Visscher, P. M., \& Wray, N. R. (2009). Harnessing the information contained within genome-wide association studies to improve individual prediction of complex disease risk. Human Molecular Genetics, 18, 3525-3531.

Fanous, A., Gardner, C. O., Prescott, C. A., Cancro, R., \& Kendler, K. S. (2002). Neuroticism, major depression and gender: A population-based twin study. Psychological Medicine, 32, 719-728.

Farmer, A., Redman, K., Harris, T., Mahmood, A., Sadler, S., Pickering, A., \& McGuffin, P. (2002). Neuroticism, extraversion, life events and depression: The Cardiff Depression Study. British Journal of Psychiatry, 181, 118-122.

Frojd, S., Kaltiala-Heino, R., \& Rimpela, M. (2007). The association of parental knowledge and family structure with 
diverse maladjustment outcomes in middle adolescent boys and girls. Nordic Journal of Psychiatry, 62, 296-303.

Frojd, S. A., Nissinen, E. S., Pelkonen, M. U., Marttunen, M. J., Koivisto, A. M., \& Kaltiala-Heino, R. (2008). Depression and school performance in middle adolescent boys and girls. Journal of Adolescence, 31, 485-498.

Gleason, K. A., Jensen-Campbell, L. A., \& Richardson, D. S. (2002). Agreeableness as a predictor of aggression in adolescence. Aggressive Behavior, 30, 43-61.

Goldner, J., Quimby, D., Richards, M., Zakaryan, A., Miller, S., Dickson, D., \& Chilson, J. (2016). Relations of parenting to adolescent externalizing and internalizing distress moderated by perception of neighborhood danger. Journal of Clinical Child and Adolescent Psychology, 45, 141154.

Goldstein, B. L., \& Klein, D. N. (2014). A review of selected candidate endophenotypes for depression. Clinical Psychology Review, 34, 417-427.

Gottesman, I., \& Gould, T. D. (2003). The endophenotype concept in psychiatry: Etymology and strategic intentions. American Journal of Psychiatry, 160, 636-645.

Hamza, C. A., \& Willoughby, T. (2011). Perceived parental monitoring, adolescent disclosure, and adolescent depressive symptoms: A longitudinal examination. Journal of Youth and Adolescence, 40, 902-915.

Horwitz, B. N., \& Neiderhiser, J. M. (2011). Geneenvironment interplay, family relationships, and child adjustment. Journal of Marriage and Family, 73, 804816.

Hyde, C. L., Nagle, M. W., Tian, C., Chen, X., Paciga, S. A., Wendland, J. R., ... Winslow, A. R. (2016). Identification of 15 genetic loci associated with risk of major depression in individuals of European descent. Nature Genetics, 48, 10311036.

Jensen-Campbell, L. A., Adams, R., Perry, D. G., Workman, K. A., Furdella, J. Q., \& Egan, S. K. (2002). Agreeableness, extraversion, and peer relations in early adolescence: Winning friends and deflecting aggression. Journal of Research in Personality, 36, 224-251.

Jensen-Campbell, L. A., \& Graziano, W. G. (2001). Agreeableness as a moderator of interpersonal conflict. Journal of Personality, 69, 323-361.

Kelly, L. M., Becker, S. J., \& Spirito, A. (2017). Parental monitoring protects against the effects of parent and adolescent depressed mood on adolescent drinking. Addictive Behaviors, 75, 7-11.

Kerr, M., \& Stattin, H. (2000). What parents know, how they know it, and several forms of adolescent adjustment: Further support for a reinterpretation of monitoring. Developmental Psychology, 36, 360-380.

Kessler, R. C., Chiu, W. T., Demler, O., \& Walters, E. E. (2005). Prevalence, severity, and comorbidity of 12 -month DSMIV disorders in the National Comorbidity Survey replication. Archives of General Psychiatry, 62, 617-627.

Klahr, A. M., \& Burt, S. A. (2014). Elucidating the etiology of individual differences in parenting: A meta-analysis of behavioral genetic research. Psychological Bulletin, 140, 544586.
Klein, D. N., Kotov, R., \& Bufferd, S. J. (2011). Personality and depression: Explanatory models and review of the evidence. Annual Review of Clinical Psychology, 7, 269-295.

Kotov, R., Gamez, W., Schmidt, F., \& Watson, D. (2010). Linking 'big' personality traits to anxiety, depressive, and substance use disorders: A meta-analysis. Psychological Bulletin, 136, 768-821.

Lac, A., \& Crano, W. D. (2009). Monitoring matters: Metaanalytic review reveals the reliable linkage of parental monitoring with adolescent marijuana use. Perspectives in Psychological Science, 4, 578-586.

Leerkes, E. M., Su, J., Calkins, S., Henrich, V. C., \& Smolen, A. (2017). Variation in mothers' arginine vasopressin receptor 1a and dopamine receptor D4 genes predicts maternal sensitivity via social cognition. Genes, Brain, and Behavior, 16, 233-240.

Lenzenweger, M. F. (2013). Thinking clearly about the endophenotype-intermediate phenotype-biomarker distinctions in developmental psychopathology research. Development and Psychopathology, 25(4 Pt 2), 1347-1357.

Li, J. J., Savage, J. E., Kendler, K. S., Hickman, M., Mahedy, L., MaCleod, J., ... Dick, D. M. (2017). Polygenic risk, personality dimensions, and adolescent alcohol use problems: A longitudinal study. Journal of Studies on Alcohol and Drugs, $78,442-451$.

Lohoff, F. W. (2010). Overview of the genetics of major depressive disorder. Current Psychiatry Reports, 12, 539-546.

MacKinnon, D. P., Lockwood, C. M., \& Williams, J. (2004). Confidence limits for the indirect effect: Distribution of the product and resampling methods. Multivariate Behavioral Research, 39, 99-128.

Martin, A. R., Gignoux, C. R., Walters, R. K., Wojcik, G. L., Neale, B. M., Gravel, S., ... Kenny, E. E. (2017). Human demographic history impacts genetic risk prediction across diverse populations. American Journal of Human Genetics, 100, 635-649.

McGuire, S., Segal, N. L., \& Hershberger, S. (2012). Parenting as phenotype: A behavioral genetic approach to understanding parenting. Parenting, Science and Practice, 12, 192-201.

Merikangas, K. R., He, J., Burstein, M., Swanson, S. A., Avenevoli, S., Cui, L., .. Swendsen, J. (2010). Lifetime prevalence of mental disorders in US adolescents: Results from the National Comorbidity Study-Adolescent Supplement (NCS-A). Journal of the American Academy of Child and Adolescent Psychiatry, 49, 980-989.

Miller, J. D., Lynam, D. R., \& Jones, S. (2008). Externalizing behavior through the lens of the five-factor model: A focus on agreeableness and conscientiousness. Journal of Personality Assessment, 90, 158-164.

Mullins, N., Power, R. A., Fisher, H. L., Hanscombe, K. B., Euesden, J., Iniesta, R., ... Lewis, C. M. (2016). Polygenic interactions with environmental adversity in the aetiology of major depressive disorder. Psychological Medicine, 46, 759770.

Neiderhiser, J. M., Reiss, D., Pedersen, N. L., Lichtenstein, P., Spotts, E. L., Hansson, K., ... Ellhammer, O. (2004). Genetic and environmental influences on mothering of 
adolescents: A comparison of two samples. Developmental Psychology, 40, 335-351.

Nock, M. K., Kazdin, A. E., Hiripi, E., \& Kessler, R. C. (2006). Prevalence, subtypes, and correlates of DSM-IV conduct disorder in the National Comorbidity Survey Replication. Psychological Medicine, 36, 699-710.

Okbay, A., Baselmans, B. M., De Neve, J. E., Turley, P., Nivard, M. G., Fontana, M. A., .. Cesarini, D. (2016). Genetic variants associated with subjective well-being, depressive symptoms, and neuroticism identified through genomewide analyses. Nature Genetics, 48, 624-633.

Peyrot, W. J., Milaneschi, Y., Abdellaoui, A., Sullivan, P. F., Hottenga, J. J., Boomsma, D. I., \& Penninx, B. W. (2014). Effect of polygenic risk scores on depression in childhood trauma. The British Journal of Psychiatry, 205, 113119.

Plomin, R. J., DeFries, J. C., \& Loehlin, J. C. (1977). Genotypeenvironment interaction and correlation in the analysis of human behavior. Psychological Bulletin, 84, 309-322.

Purcell, S., Neale, B., Todd-Brown, K., Thomas, L., Ferreira, M. A. R., Bender, D., ... Sham, P. C. (2007). PLINK: A tool set for whole-genome association and population-based linkage analyses. American Journal of Human Genetics, 81, 559-575.

Racz, S. J., \& McMahon, R. J. (2011). The relationship between parental knowledge and monitoring and child and adolescent conduct problems: A 10-year update. Clinical Child and Family Psychology Review, 14, 377-398.

Rhew, I. C., Fleming, C. B., Vander Stoep, A., Nicodimos, S., Zheng, C., \& McCauley, E. (2017). Examination of cumulative effects of early adolescent depression on cannabis and alcohol use disorder in late adolescence in a communitybased cohort. Addiction, 112, 1952-1960.

Roberts, B. W., Caspi, A., \& Moffitt, T. E. (2001). The kids are alright: Growth and stability in personality development from adolescence to adulthood. Journal of Personality and Social Psychology, 81, 670-683.

Roberts, B. W., Walton, K. E., \& Viechtbauer, W. (2006). Patterns of mean-level change in personality traits across the life course: A meta-analysis of longitudinal studies. Psychological Bulletin, 132, 1-25.

Rohde, P., Lewinsohn, P. M., Klein, D. N., Seeley, J. R., \& Gau, J. M. (2013). Key Characteristics of major depressive disor- der occurring in childhood, adolescence, emerging adulthood, adulthood. Clinical Psychological Science, 1, 41-53.

Salvatore, J. E., Aliev, F., Bucholz, K., Agrawal, A., Hesselbrock, V., Hesselbrock, M., ... Dick, D. M. (2015). Polygenic risk for externalizing disorders: Gene-by-development and gene-by-environment effects in adolescents and young adults. Clinical Psychological Science, 3, 189-201.

Samek, D. R., Wilson, S., McGue, M., \& Iacono, W. G. (2016). Genetic and environmental influences on parent-child conflict and child depression through late adolescence. Journal of Clinical Child and Adolescent Psychology, 1-16.

Scarr, S., \& McCartney, K. (1983). How do people make their own environment? A theory of gene-environment effects. Child Development, 54, 424-435.

Schofield, T. J., Conger, R. D., Donnellan, M. B., Jochem, R., Widaman, K. F., \& Conger, K. J. (2012). Parent personality and positive parenting as predictors of positive adolescent personality development over time. Merrill-Palmer Quarterly, 58, 255-283.

Small, D. M., Simons, A. D., Yovanoff, P., Silva, S. G., Lewis, C. C., Murakami, J. L., \& March, J. (2008). Depressed adolescents and comorbid psychiatric disorders: Are there differences in the presentation of depression? Journal of $A b$ normal Child Psychology, 36, 1015-1028.

Subbarao, A., Rhee, S. H., Young, S. E., Ehringer, M. A., Corley, R. C., \& Hewitt, J. K. (2008). Common genetic and environmental influences on major depressive disorder and conduct disorder. Journal of Abnormal Psychology, 36, 433444.

Van IJzendoorn, M. H., Bakermans-Kranenburg, M. J., \& Mesman, J. (2008). Dopamine system genes associated with parenting in the context of daily hassles. Genes, Brain and Behavior, 7, 403-410.

Vukasovic, T., \& Bratko, D. (2015). Heritability of personality: A meta-analysis of behavior genetic studies. Psychological Bulletin, 141, 769-785.

Watson, D., Stasik, S. M., Ellickson-Larew, S., \& Stanton, K. (2015). Extraversion and psychopathology: A facet-level analysis. Journal of Abnormal Psychology, 124, 432-446.

Wolff, J. C., \& Ollendick, T. H. (2006). The comorbidity of conduct problems and depression in childhood and adolescence. Clinical Child and Family Psychology Review, 9, 201-220. 